\title{
Optimization for framework design of new product introduction management system
}

\author{
Ma Ying, Wu Hongcui \\ Tianjin Electronic Information Vocational Technology College, 300350
}

Keywords: the new product introduction; critical path; static project

\begin{abstract}
.in the optimization process of framework design of new product introduction management system, using the current algorithm for framework optimization design, communication of materials and parts information has certain deviation, results in longer introduction time of new product. A framework optimization design method of new product introduction management system is proposed based on critical path algorithm. The method carries on the detailed analysis for the new product introduction system, and new product introduction management process model is built on this basis to depict the network diagram of new product introduction management process nodes, afterwards, using the critical path algorithm to calculate the critical path of framework design for new product introduction management system, the management of new products introduction is divided into four stages, combined with management style of static project to optimize the design for framework of new product introduction management system. The experiments show that the framework optimization design method for new product introduction management system based on critical path algorithm have good performance on designing, and high precision.
\end{abstract}

\section{Introduction}

At present, in the domestic and international market competition, the substantial enterprises in various field is getting rid of stale and bring forth the fresh 1.2.3. In this case, the design for new product introduction management system has become the core tasks, attracted a lot of experts and scholars' attention 4.5.6. Due to the far-reaching developing significance of framework optimization design of new products introduction management system, so it has become a focus of research $7 \sim 10$.

Currently, the mainstream framework optimization design method including the method based on ant colony algorithm, based on the particle algorithm, and based on neural network algorithm. But the above method has certain deviation, and lead to the problem of longer time in new product introducing process.

In view of the above problems, a framework optimization design method of new product introduction management system is proposed based on critical path algorithm. The design method has high accuracy and good performance.

\section{Analysis of new product introduction management system}

To build a good new product introduction management system should have the following factors:

(1) in framework optimization design of new product introduction management system, importance of corporate culture on teamwork and employee communication have to be increased.

(2) in framework optimization design of new product introduction management system, the organizational structure of enterprises has to be completed.

(3) in framework optimization design of new product introduction management system, R \& D and manufacturing center have to be established.

(4) in framework optimization design of new product introduction management system, new product introduction cycle has to be regulated.

(5) in framework optimization design of new product introduction management system, the number of material of new product has to be constrained. 
(6) in framework optimization design of new product introduction management system, the type of new products has to be defined.

(7) in framework optimization design of new product introduction management system, the introduction number of new product has to be planned.

\section{The proposal and implementation of framework optimization design method of new product introduction management system.}

\subsection{The establishment of new product introduction management process model}

The introduction process model of new product contains contents like clear theme, research activities, raise problem solutions, develop prototype development and implement solutions to problems and etc.. The following factors need to be considered when forming the new product introduction management process model: the origin of idea and concept design, the accurate definition and determination for new product, focus on new product development and prototyping, establishment of the commercialized management system.

New product introduction management can be divided into four stages. The specific steps are as follows in detail:

The workflow is represented by $W$, and the framework design stage of new product introduction management system can be expressed as $A_{w}=\left\{A_{1}, A_{2}, A_{3}, A_{4}\right\}, A$ represents stage work during framework designing process of new product introduction management system. The directed edge set of new product introduction process node network graph can be calculated with the following formula:

$$
F_{W}=\left[F_{1}, F_{2}, F_{3}, F_{4} \ldots\right]
$$

In framework optimization design of new product introduction management system, the formation of new product introduction management process model is defined as a $M / M / 1$ network, where each activity is a $M / M / 1$ system, integrated in the critical path algorithm to calculate the time cycle used by new product introduction management process. Expressed by the following equation:

$$
\begin{aligned}
& W=\max \left(W 1, W 2, \cdots, W_{n}\right)= \\
& \max \left(\sum_{j=1}^{n} w_{j}\right)=\max \left(\sum_{j=1}^{n} \frac{1}{\mu_{J}-\lambda_{i}}\right)
\end{aligned}
$$

Based on the above equation to obtain the critical path of framework design of new product management system, stated by the following equation;

$$
\begin{aligned}
& W_{k}=\max \left(W_{1}, W_{2}, \cdots, W_{n}\right)= \\
& \max \left(\sum_{j=1}^{n} w_{j}\right)=\max \left(\sum_{j=1}^{n} \frac{1}{\mu_{J}-\lambda / P_{i}}\right)
\end{aligned}
$$

In framework optimization design of new product introduction management system, the optimization design critical path obtained from above equation is adopted to divide new product introduction process into four stages:

(1) determine the critical path. According to the functional model of new product introduction process management, to establish the node network graph, network graph shows the estimated completion duration of each activity, the duration of an activity is assessed based on the most likely time probability analysis, and the total duration is the expectation required by customers, usually 100 days. Expressed by equation:

$$
T=\frac{1}{\mu-\lambda}
$$

(2) analysis and diagnosis of the critical path. In framework optimization design of new product introduction management system,, the critical paths is utilized to analyze the key events on the 
critical path in depth, figure out problems and correct in a timely manner.

(3) reengineering for the new product introduction management process. In framework optimization design of new product introduction management system, based on appreciation and non-appreciation, time consumption, major and minor principles, referring and using the critical path method to diagnose and analyze new product management process.

(4) the transformation of the new product introduction process management. Evaluating from the time period, the time period before the management process reengineering is 102 days, if unexpected events occur, extremely easy to cause the delay of new product introduction, on the basis of critical path method and increased value-added operations to calculate the cycle time of new process rafter eengineering, the process will be 21 days in advance.

3.2 The implementation of framework optimization design method of the new product introduction management system.

Nowadays, the framework optimization design method of new product introduction management system, mainly including the following two types: static project and dynamic project. In order to ensure the long-term effect of framework optimization design method of new product introduction management system, according to the characteristics of the new product introduction management system, the framework of new product introduction management system is optimized with the management style integrated with static project. The specific steps are as follows in detail:

(1)strengthen the training and participation of the staff.

(2)designate principals for new product introduction management and corresponding confirmation form in various departments.

(3)Organize new product introduction management supervision team.

\section{Simulation results}

In order to prove that the effectiveness of the proposed framework optimization design method of new product introduction management system based on critical path algorithm, experiments are needed. Building framework optimization design virtual simulation platform of new product introduction management system in Windows2003 environment, using the traditional algorithm and the improved algorithm for new product management system framework design, design task completion time of two algorithms were compared to verify the design performance of different algorithms. The results were in Table 1 and table 2 .

Table 1 Completion time of framework optimization design task of new product introduction management system with the traditional algorithm

\begin{tabular}{cccc}
\hline NO. & Task code & Task name & $\begin{array}{c}\text { Task completion } \\
\text { time (days) }\end{array}$ \\
\hline 1 & A & $\begin{array}{c}\text { Obtain the market } \\
\text { demand } \\
\text { Develop product } \\
\text { proposal }\end{array}$ & 60 \\
2 & B & $\begin{array}{c}\text { Design planning } \\
\text { book }\end{array}$ & 20 \\
3 & C & $\begin{array}{c}\text { Collect the test data } \\
\text { Put into production }\end{array}$ & 30 \\
4 & D & 47 \\
\hline
\end{tabular}


Table 2 Completion time of framework optimization design task of new product introduction management system with the proposed algorithm

\begin{tabular}{cccc}
\hline NO. & Task code & Task name & $\begin{array}{c}\text { Task completion } \\
\text { time (days) }\end{array}$ \\
\hline 1 & A & $\begin{array}{c}\text { Obtain the market } \\
\text { demand } \\
\text { Develop product } \\
\text { proposal }\end{array}$ & 30 \\
2 & B & Design planning book & 15 \\
3 & C & Collect the test data & 15 \\
4 & D & Put into production & 20 \\
5 & E & D & \\
\hline
\end{tabular}

Seen from Tables 1 and 2, the task completion time of improved algorithm was significantly lower than the traditional algorithm. Mainly because The method carries on the detailed analysis for the new product introduction system, and new product introduction management process model is built on this basis to depict the network diagram of new product introduction management process nodes, afterwards, using the critical path algorithm to calculate the critical path of framework design for new product introduction management system, so as to ensure the time efficiency of new product introduction.

\section{Conclusion}

During the optimization process of framework design of new product introduction management system, using the current algorithm for framework optimization design, communication of materials and parts information has certain deviation, results in longer introduction time of new product. A framework optimization design method of new product introduction management system is proposed based on critical path algorithm. The method carries on the detailed analysis for the new product introduction system, and new product introduction management process model is built on this basis to depict the network diagram of new product introduction management process nodes, afterwards, using the critical path algorithm to calculate the critical path of framework design for new product introduction management system, the management of new products introduction is divided into four stages, combined with management style of static project to optimize the design for framework of new product introduction management system. The experiments show that the framework optimization design method for new product introduction management system based on critical path algorithm have good performance on designing, and high precision.

\section{References}

[1] Rong Linan, Wang Jian. Analysis of the introduction of culture teaching in teacher's role in ESL framework based on [J]. Journal of Liaoning Medical University: Social Science Edition, 2014, 12 (1): 77-79.

[2] Cao Xin. Enterprise integrated control equipment assets theory (ACCM) framework and key points of [J]. China import equipment engineering, 2013, (4): 29-31.

[3] Zhou Shumei (3). The teaching management system of colleges and universities in the design and implementation of [J]. China school education management, 2013, (18): 52-52.

[4] Gao Weiwei, Liu Guangmin and so on. And the realization and application of [J]. software, computer design of university personnel salary management system 2013 (13): 258-258.

[5] Wang Xueqing, Cheng Qifeng. The office management system in design of UML based on [J]. software, 2013, (11): 36-39.

[6] Du Qinsheng, Tang Yonglin, Yang Huapeng. Design and implementation of [J]. Journal of Changchun University performance management system of teachers in Colleges and Universities: 
Natural Science Edition, 2013, (10): 1249-1251.

[7] Wu Kaixing, Chen Xu, Zhai ziyong. And the realization of [J]. computer applications and software, the design of management system of resident health record 2013, 30 (4): 214-216.

[8] The gang, Luo Qi. With the implementation of [J]. technology vision, design automation of warehouse management system 2013 (2): 27-28.

[9] Dong Zhe, Lin Junwei, Sun Dehui. Technology and application, the design and application of the [J]. NET automation of warehouse management system based on 2013, 32 (1): 14-19.

[10] Du Qin, Ni Jun, Fan Zhuo. Design and Simulation of management system of sports venues of computer simulation for [J]. based on VR technology, 2013, 30 (3): 356-359. 